\title{
Predicting the effect of dissolved humic acid on sorption of benzotriazole to biochar
}

\author{
Lin $\mathrm{Wu}^{1,2} \cdot$ Xiaoli Zhao ${ }^{1} \cdot$ Erping $\mathrm{Bi}^{1}$ (D)
}

Received: 13 August 2021 / Accepted: 30 November 2021

(c) The Author(s) 2022

\begin{abstract}
Dissolved organic matter plays a critical role in affecting sorption properties of biochar for organic contaminants. In this study, dissolved humic acid (DHA) as a representative of dissolved organic matter and oak sawdust-derived biochar as a sorbent were prepared and characterized. Roles of DHA in sorption of benzotriazole (BTA), an emerging organic contaminant, to biochar in different electrolyte solutions were investigated. The results revealed the dual roles of DHA in BTA sorption to biochar. On the one hand, DHA can compete for sites and/or block pores available for BTA to inhibit the adsorption of BTA to biochar. On the other hand, the sorbed DHA on biochar can serve as additional partitioning phase to promote the partition of BTA. The finding was in accordance with the site energy distribution analysis of BTA sorption that the site energy of the highest occurring frequency in the DHA-BTA system was lower than that in the DHA-free system (3.41-10.4 versus 13.1-20.1 kJ mol${ }^{-1}$ ). The variation in apparent BTA sorption to biochar affected by DHA was thus a combination of changes in both its partition and adsorption properties. A modified Dual-mode model including the aqueous concentration of DHA was proposed to predict the effect of DHA on BTA sorption to biochar in different electrolyte solutions, which showed good prediction performance with most BTA sorption coefficient $\left(K_{d}, \mathrm{~L} \mathrm{~g}^{-1}\right)$ deviations within $0.1 \log$ unit.
\end{abstract}

\section{Highlights}

- A model was proposed to predict the effect of dissolved humic acid (DHA) on benzotriazole (BTA) sorption to biochar.

- The dual roles of DHA in promoting partition but inhibiting adsorption of BTA to biochar were revealed.

Erping Bi

bi@cugb.edu.cn

Lin Wu

wulin_shs@mail.cgs.gov.cn

Xiaoli Zhao

xlzhaony@163.com

1 School of Water Resources and Environment, Beijing Key Laboratory of Water Resources and Environmental Engineering, and MOE Key Laboratory of Groundwater Circulation and Environmental Evolution, China University of Geosciences (Beijing), Beijing 100083, China

2 China Geological Survey (CGS) \& Hebei Province Key Laboratory of Groundwater Contamination and Remediation, Institute of Hydrogeology and Environmental

Geology, Chinese Academy of Geological Sciences, Shijiazhuang 050061, China
- Site energy distribution was introduced to verify the roles of DHA in BTA sorption to biochar. 
Keywords Emerging organic contaminant $\cdot$ Black carbon $\cdot$ Dissolved organic matter $\cdot$ Sorption $\cdot$ Site energy distribution

\section{Introduction}

Benzotriazole (BTA), one kind of important industrial auxiliary chemicals, is widely used as a corrosion inhibitor, UV absorber, antifreeze and antifogging agent (Wagner et al. 2020). More recently, BTA was regarded as a class of emerging environmental organic contaminants, and it has been detected in municipal wastewater, surface water, groundwater, soils and sediments at levels ranging from ng $\mathrm{L}^{-1}$ to $\mathrm{mg} \mathrm{L}^{-1}$ or from $\mathrm{ng} \mathrm{kg}^{-1}$ to $\mathrm{mg} \mathrm{kg}^{-1}$ (Kahle et al. 2009; Kowalska et al. 2019; Zhang et al. 2011). Due to its omnipresence, BTA was proposed to be an indicator of anthropogenic contamination in the environment (Kahle et al. 2009; Shi et al. 2019). Since BTA can cause potential hazards to microorganisms, fish, plants, invertebrates and terrestrial mammals in different environmental media and induce long-term negative effects in ecosystems (Alotaibi et al. 2015; He et al. 2019; Liang et al. 2017), it has aroused a widespread public concern.

Sorption is a critical process influencing the transport, degradation and bioavailability of contaminants in the environment. Previous research has focused on the sorption of BTA to soils, peat, compost and metal oxides. Specifically, sorption of BTA to peat and compost with a high organic carbon content was greater than that to various soil matrices, suggesting the dominant role of soil organic matter rather than materials in controlling BTA sorption (Breedveld et al. 2003; Jia et al. 2007). Sorption mechanisms of BTA were proposed to involve some specific interactions (such as $\pi-\pi$ interactions and hydrogen bonding) according to the type of sorbent (Hart et al. 2004; Xu et al. 2010). Furthermore, the variation in solution chemistry (such as $\mathrm{pH}$ and ionic type) would alter BTA sorption mechanisms by affecting speciation of BTA and surface properties of sorbent (Bi et al. 2007; Xing et al. 2016), which should be paid more attention to in the prediction of its environmental behaviors. However, reports on sorption of BTA to biochar, an important component of the soil carbon pool, are rare.

Biochar is produced from pyrolysis of different biomass wastes under oxygen-limited conditions. The use of biochar has attracted increasing interest as a sustainable means of improving soil quality, carbon sequestration and pollution control (El-Naggar et al. 2019; Jin et al. 2016; Wu et al. 2020b). Recent studies showed that biochar can contribute considerably to the overall sorption of organic compounds in soil on account of its higher sorption capacity compared to some natural organic matter (Accardi-Dey and Gschwend 2003). The nature and sorption properties of biochar are mainly controlled by the pyrolysis temperature. Increase in pyrolysis temperature often results in the enhanced surface area, pore volume and carbonized fraction of biochar (Keiluweit et al. 2010; Xiao et al. 2014). Sorption mechanisms of biochar for neutral aromatic compounds changed from absorption-dominant (i.e., partition-dominant) at low pyrolysis temperatures to adsorption-dominant at higher pyrolysis temperatures (Chen et al. 2012; Lu et al. 2020). Usually, the adsorption of aromatic compounds to biochar is driven by $\pi-\pi$ interactions, along with a pore filling mechanism (Kah et al. 2017; Keiluweit and Kleber 2009).

New evidence presents the fact that the sorptive activities of biochar for organic contaminants can be strongly influenced by coexisting dissolved organic matter (DOM) in the environment (Zhang et al. 2015). It was found that DOM reduced sorption of organic contaminants to carbonaceous materials via competition or hindering the accessibility of adsorption sites (Engel and Chefetz 2016; Wu et al. 2018a). In addition, the competition may vary under different conditions since sorption of DOM to solid phase can be affected by different types of cations (Kalbitz et al. 2000). On the contrary, DOM sorption can modify the surface characteristics of sorbent and provide additional active sites, leading to enhanced sorption of organic compounds (Lian et al. 2015; Wu et al. 2018b, 2020a). So far, it remains difficult to draw a proper conclusion on the roles of DOM in sorption of organic contaminants including BTA because of the observed coexisting enhancing and suppressing effects. Specifically, biochar sorption for BTA can involve both partition and adsorption due to the mixtures of noncarbonized and carbonized phase in sorbent (Xing et al. 2016). The effect of DOM on sorption of BTA to biochar would come from that on both partition and adsorption, which may be quite different due to the diverse roles of DOM. Furthermore, site energy distribution analysis was often used to describe sorption on heterogeneous surfaces (Li et al. 2021; Shen et al. 2015). Estimation of site energy distribution of sorbent for target organic compounds as affected by DOM might be helpful for the analysis of sorption mechanism from the standpoint of energy.

The aims of the present study are to demonstrate the hypothesis that the effect of DOM on sorption of BTA to biochar comes from its diverse roles in partition and adsorption, and develop a quantitative approach to predict the effect of DOM on BTA sorption. To this end, DOM and biochar were prepared and characterized. Batch experiments on DOM and BTA sorption to biochar, and dialysis bag experiments on BTA sorption to DOM in deionized water, $\mathrm{NaCl}$ and $\mathrm{CaCl}_{2}$ solutions were conducted. The site energy distribution was determined to analyze the interactions between BTA and biochar as affected by DOM. Based on the characterization, experimental results and site energy distribution 
analysis, the effect of DOM on BTA sorption to biochar was elucidated. A model to predict the DOM effect on BTA sorption was also proposed.

\section{Materials and methods}

\subsection{Chemicals}

BTA $\left(\mathrm{C}_{6} \mathrm{H}_{5} \mathrm{~N}_{3},>99 \%\right.$ purity, CAS number: $\left.95-14-7\right)$ was bought from Dr. Ehrenstorfer GmbH (Augsburg, Germany). It has an octanol-water partition coefficient $\left(\log K_{\text {ow }}\right)$ of 1.44 (SRC PhysProp Database), a $\mathrm{p} K_{\mathrm{a}}$ of 8.6 (estimated by MarvinSketch 16.2.22) and a water solubility of $19.8 \mathrm{~g}$ $\mathrm{L}^{-1}$. Stock solution of BTA was prepared by dissolving it in methanol (HPLC grade) obtained from Tedia Company (USA). $\mathrm{HCl}, \mathrm{NaOH}, \mathrm{NaCl}$ and $\mathrm{CaCl}_{2}$ were purchased from Beijing Chemical Works (China). Deionized water was used in all experiments.

\subsection{Preparation and characterization of biochar and DOM}

The biochar was produced from oak sawdust by slow pyrolysis. Briefly, the oak sawdust was sealed in a crucible and then put into a muffle furnace. The carbonization temperature was raised to $500{ }^{\circ} \mathrm{C}$ at a speed of $5{ }^{\circ} \mathrm{C} \mathrm{min}{ }^{-1}$ and held for $6 \mathrm{~h}$. After $6 \mathrm{~h}$ of heating, the furnace was turned off and the sample was allowed to cool naturally to room temperature. Then, the carbonized samples were treated in $1.0 \mathrm{M}$ $\mathrm{HCl}$ for $24 \mathrm{~h}$ to remove the minerals. Following a thorough washing with deionized water until to neutral $\mathrm{pH}$, to remove dissoluble matter at the same time, the residues were dried at $110{ }^{\circ} \mathrm{C}$ for $24 \mathrm{~h}$ to obtain biochar. The generated biochar was ground and sieved through a 100-mesh screen and then stored in a glass jar at room temperature $\left(25 \pm 1{ }^{\circ} \mathrm{C}\right)$.

Humic acid, a representative of natural organic matter possessing aromatic rings substituted with hydroxyl and carboxyl groups, was used to prepare DOM (Li et al. 2019). Humic acid $(49.8 \% \mathrm{C}, 3.13 \% \mathrm{H}, 1.32 \% \mathrm{~N}$ and $25.2 \% \mathrm{O})$ was bought from Sinopharm Chemical Reagent Co., Ltd. (China). Dissolved humic acid (DHA) was prepared using a modified method (Wu et al. 2020a). In brief, a mass of $5 \mathrm{~g}$ of humic acid was dissolved in one liter of deionized water. The mixture was stirred with a glass rod to facilitate dissolution. The supernatant was collected and filtered through a $0.45 \mu \mathrm{m}$ sterilized membrane. The extract was stored at $4{ }^{\circ} \mathrm{C}$ and diluted to desired concentrations for subsequent experiments.

The contents of $\mathrm{C}, \mathrm{H}, \mathrm{O}$ and $\mathrm{N}$ of biochar were analyzed by an Elementar vario EL (Germany). Ash content was determined as described previously (Chen et al. 2012). The biochar sample was placed in an open-top crucible in a muffle furnace. The temperature was raised to $800{ }^{\circ} \mathrm{C}$ at $5{ }^{\circ} \mathrm{C} \mathrm{min}{ }^{-1}$ and then held for $4 \mathrm{~h}$. The furnace was allowed to cool to room temperature. Ash referred to the residue remaining in the crucible. The functional groups of biochar were characterized by Fourier transform infrared (FTIR) spectral analysis in the $4000-600 \mathrm{~cm}^{-1}$ region using a Thermo Electron NeXus 8700 Spectrophotometer (Waltham, MA, USA) with pressed $\mathrm{KBr}$ pellets. The specific surface area and pore size distribution of biochar were measured with $\mathrm{N}_{2}$ adsorption/desorption at liquid nitrogen temperature by a Micromeritics ASAP $2020 \mathrm{M}+\mathrm{C}$ (USA). The $\mathrm{pH}$ value at the point of zero charge $\left(\mathrm{pH}_{\mathrm{pzc}}\right)$ of biochar was determined by the $\mathrm{pH}$ drift method (Yang et al. 2004). Briefly, a mass of $0.06 \mathrm{~g}$ of biochar was added to $20 \mathrm{~mL}$ of $0.005 \mathrm{M} \mathrm{CaCl}_{2}$ solution with $\mathrm{pH}$ ranging from 2.0 to 8.0 in sealed brown glass vials. The vials were shaken at $175 \mathrm{rpm}$ for $24 \mathrm{~h}$ to reach equilibrium. Then, the final $\mathrm{pH}$ of the solution was determined and compared with the initial $\mathrm{pH}$. The $\mathrm{pH}_{\mathrm{pzc}}$ was identified as the $\mathrm{pH}$ value at the intersection of a measured curve with the $\mathrm{pH}_{\text {final }}=\mathrm{pH}_{\text {initial }}$ line.

The size distribution of DHA was determined via dynamic light scattering (DLS, Brookhaven ZetaPALS instrument) with an angle of 90 degree to the incident beam. The conformation of DHA was characterized by atomic force microscopy (AFM, Scanning Peak Microscopy, Dimension Icon, Bruker, USA) with a scanning speed of $1.00 \mathrm{~Hz}$. The charge properties of DHA were evaluated by a Nano-Z Zetasizer (Malvern Instruments Ltd.) at different $\mathrm{pH}$ values (3.0-9.0). The concentration of DHA was $30 \mathrm{mg}$ $\mathrm{L}^{-1}$ in the characterization.

\subsection{Sorption experiments}

Sorption of DHA to biochar was investigated by performing batch sorption experiments. A mass of $0.03 \mathrm{~g}$ biochar was placed in $40 \mathrm{~mL}$ brown glass bottles and then $30 \mathrm{~mL}$ of sorbate solution was added. The solid/liquid ratio was chosen to reach approximately $20-80 \%$ removal rate of sorbate through pre-experiments. The concentrations of DHA in different solutions (deionized water, $0.01 \mathrm{M} \mathrm{NaCl}$ or $0.01 \mathrm{M} \mathrm{CaCl}_{2}$ background electrolyte) ranged from 0 to $14 \mathrm{mg} \cdot \mathrm{L}^{-1}$. The initial solution $\mathrm{pH}$ was adjusted to $6.0 \pm 0.1$ with $0.1 \mathrm{M} \mathrm{HCl}$ and/or $\mathrm{NaOH}$. The samples were shaken in a horizontal thermostatic shaker (QYC2112) at $175 \mathrm{rpm}$ and $25 \pm 1{ }^{\circ} \mathrm{C}$ in the dark to achieve sufficient reaction as described previously (Wu et al. 2020a) and held for $3 \mathrm{~d}$ to reach sorption equilibrium. Subsequently, the samples were centrifuged (3000 rpm for $10 \mathrm{~min}$ ) and the supernatants were filtered through a $0.45 \mu \mathrm{m}$ sterilized membrane. Finally, the aqueous DHA concentration was determined. All experiments were performed in duplicate. No obvious solute loss $(<2 \%)$ by the sterilized membrane 
was detected. Blanks without biochar were set to determine the minor loss $(<1 \%)$ of solute in degradation or sorption by glass bottles.

Sorption of BTA to DHA was measured through dialysis bag experiments (Li et al. 2019). Before use, the dialysis bags (100 Da, Union Carbide Corporation, USA) were boiled and rinsed with ethanol and deionized water to remove surface impurities. The average molecular weight of DHA used in this study was determined to be 919 Da by gel permeation chromatography. Thus, sorption of BTA to DHA fractions with molecular weight less than $100 \mathrm{Da}$ can be neglected. A volume of $10 \mathrm{~mL}$ of various solutions (deionized water, $0.01 \mathrm{M} \mathrm{NaCl}$ or $0.01 \mathrm{M} \mathrm{CaCl}_{2}$ background electrolyte) containing $8.1 \mathrm{mg} \mathrm{L}^{-1} \mathrm{DHA}$ was added into dialysis bags. The dialysis bags were put in 40-mL brown glass bottles. A volume of $20 \mathrm{~mL}$ of various solutions containing $30 \mathrm{mg} \mathrm{L}^{-1}$ BTA was added to the bottles. The initial solution $\mathrm{pH}$ was adjusted to $6.0 \pm 0.1$ by $0.1 \mathrm{M} \mathrm{HCl}$ and/or $\mathrm{NaOH}$. The bottles were placed in a horizontal thermostatic shaker at $175 \mathrm{rpm}$ and $25 \pm 1{ }^{\circ} \mathrm{C}$ in the dark for 4 days to reach equilibrium. The concentration of DHA-bound BTA was determined by the concentration difference between inside and outside of the dialysis bags (Pan et al. 2008). Negligible $(<1 \%)$ sorption of BTA to the dialysis bags was observed.

Sorption of BTA to biochar was studied by the abovedescribed batch equilibrium method with a solid/liquid ratio same as that of DHA sorption to biochar in order to make their sorption results comparable and facilitate the analysis. The initial BTA concentrations in various solutions (deionized water, $0.01 \mathrm{M} \mathrm{NaCl}$ or $0.01 \mathrm{M} \mathrm{CaCl}_{2}$ background electrolyte) with and without DHA (5.6 or $8.1 \mathrm{mg} \cdot \mathrm{L}^{-1}$ ) were in a range from 20 to $100 \mathrm{mg} \cdot \mathrm{L}^{-1}$. The volume of methanol in solution was kept within $0.1 \%$ (v/v) to avoid organic solvent effects. The initial solution $\mathrm{pH}$ was adjusted to $6.0 \pm 0.1$ with $0.1 \mathrm{M} \mathrm{HCl}$ and/or $\mathrm{NaOH}$. The samples were placed in a thermostatic shaker $\left(175 \mathrm{rpm}, 25 \pm 1^{\circ} \mathrm{C}\right)$ for 4 days to reach sorption equilibrium. The following procedures were the same as those of sorption experiments of DHA to biochar.

\subsection{Analytical methods}

BTA was determined by a high performance liquid chromatography (HPLC, Shimadzu Corp, LC-20AT, Japan) with an Eclipse XDB-C18 column (150 $\mathrm{mm} \times 4.6 \mathrm{~mm}$, Agilent) and UV detector at $254 \mathrm{~nm}$. The mobile phase was methanol-water (50:50, v:v) with a flow rate of $0.7 \mathrm{~mL} \cdot \mathrm{min}^{-1}$. The concentration of DHA was determined by a total organic carbon analyzer (Shimadzu model 3210, Japan) in the form of dissolved organic carbon. The amount of $\mathrm{NaOH}$ used in adjusting solution $\mathrm{pH}$ was monitored by measuring $\mathrm{Na}^{+}$ using ion chromatography (Dionex DX-120).

\subsection{Data analysis}

DHA sorption data were fitted by the Initial Mass isotherm (Eq. (1)) as suggested by the previous research (Nodvin et al. 1986).

$Q_{s}=m X_{i}-r$

where $Q_{s}\left(\mathrm{mg} \mathrm{g}^{-1}\right)$ is the amount of substance released to (negative value) or removed from (positive value) the solution concerning the mass of biochar, $m$ is the regression coefficient denoting the fraction of substance sorbed by biochar, $X_{\mathrm{i}}\left(\mathrm{mg} \mathrm{g}^{-1}\right)$ is the initial amount of substance in solution concerning the mass of biochar and $r\left(\mathrm{mg} \mathrm{g}^{-1}\right)$ is the intercept of the Initial Mass isotherm.

Sorption coefficient $\left(K_{d}, \mathrm{~L} \mathrm{~g}^{-1}\right)$ of DHA by biochar was calculated following Eq. (2):

$K_{d}=(m /(1-m)) V / M$

where $V(\mathrm{~L})$ is the volume of solution and $M(\mathrm{~g})$ is the mass of biochar.

Sorption isotherms of BTA were fitted by the Dualmode model (Eq. (3)), Freundlich model (Eq. (4)) and Dubinin-Astakhov model (Eq. (5)):

$C_{s}=K_{p} C_{e}+Q_{m} K_{L} C_{e} /\left(1+K_{L} C_{e}\right)$

$C_{s}=K_{F} C_{e}^{n}$

$\log C_{s}=\log Q_{m}-\left(\left(R T \ln \left(S_{w} / C_{e}\right)\right) / E\right)^{v}$

where $C_{s}\left(\mathrm{mg} \mathrm{g}^{-1}\right)$ and $C_{e}\left(\mathrm{mg} \mathrm{L}^{-1}\right)$ are equilibrium solid-phase and aqueous-phase concentrations of solute, respectively, $K_{p}\left(\mathrm{~L} \mathrm{~g}^{-1}\right)$ is the partition domain coefficient, $Q_{m}\left(\mathrm{mg} \mathrm{g}^{-1}\right)$ is the maximum adsorption capacity, $K_{L}(\mathrm{~L}$ $\left.\mathrm{mg}^{-1}\right)$ is the affinity constant, $K_{F}\left(\mathrm{mg}^{(1-n)} \mathrm{L}^{n} \mathrm{~g}^{-1}\right)$ is the affinity coefficient, $n$ is the exponent indicating the nonlinearity of sorption, $R\left(8.314 \times 10^{-3} \mathrm{~kJ} \mathrm{~mol}^{-1} \mathrm{~K}^{-1}\right)$ is the universal gas constant, $T(\mathrm{~K})$ is the absolute temperature, $S_{w}\left(\mathrm{mg} \mathrm{L}^{-1}\right)$ is the solubility of solute, $E\left(\mathrm{~kJ} \mathrm{~mol}^{-1}\right)$ is the correlating divisor and $v$ is a fitting parameter.

$K_{d}$ of BTA by biochar was calculated by Eq. (6):

$K_{d}=C_{s} / C_{e}$

The sorption coefficient of BTA to DHA $\left(K_{\text {DHA }}, \mathrm{L} \mathrm{g}^{-1}\right)$ was calculated by Eq. (7):

$K_{\mathrm{DHA}}=10^{3}\left(C_{b} / C_{f}\right) / C_{\mathrm{DHA}}$

where $C_{b}\left(\mathrm{mg} \mathrm{L}^{-1}\right)$ and $C_{f}\left(\mathrm{mg} \mathrm{L}^{-1}\right)$ are the concentrations of DHA-bound BTA and freely-dissolved BTA, respectively, and $C_{\mathrm{DHA}}\left(\mathrm{mg} \mathrm{L}^{-1}\right)$ is the aqueous DHA concentration. 
The site energy distribution $\left[F\left(E^{*}\right)\right]$ was gained by differentiating $C_{s}\left(E^{*}\right)$ to $E^{*}$ (Carter et al. 1995).

$F(E *)=-\mathrm{d} C_{s}(E *) / \mathrm{d} E *$

where $E^{*}\left(\mathrm{~kJ} \mathrm{~mol}^{-1}\right)$ is the sorption energy difference at $C_{e}$ and $S_{w^{*}}$.

The correlation between the sorption energy and $C_{e}$ can be calculated as:

$C_{e}=S_{w} \exp (-E * / R T)$

Therefore, Eq. (10) was derived from Eqs. (5), (8) and (9) as suggested by previous studies (Shen et al. 2015; Zhang et al. 2012).

$F(E *)=\ln (10) v E^{-v} E *^{v-1} 10^{\left(\log Q_{m}-E^{-v} E *^{v}\right)}$

\section{Results and discussion}

\subsection{Characteristics of biochar and DHA}

The contents of $\mathrm{C}, \mathrm{H}, \mathrm{N}, \mathrm{O}$ and ash in biochar were 73.88 , $2.77,1.99,16.95$ and $3.28 \%$, respectively. The H/C and $\mathrm{O} / \mathrm{C}$ ratios of biochar were 0.45 and 0.17 , respectively. The results indicated that the biochar still contained a certain amount of plant organic residues with plenty of oxygencontaining functional groups. FTIR spectra indicated that the peak observed at $3422 \mathrm{~cm}^{-1}$ was attributed to the $\mathrm{O}-\mathrm{H}$ stretching vibration (Fig. 1a). The asymmetric C-H stretching $\left(3065 \mathrm{~cm}^{-1}\right)$ indicated the aliphatic functional groups. The peak at $1597 \mathrm{~cm}^{-1}$ was ascribed to aromatic $\mathrm{C}=\mathrm{C}$ stretching and $\mathrm{C}=\mathrm{O}$ stretching of conjugated ketones and quinones. The band of $\mathrm{C}=\mathrm{O}$ in carboxyl and ester groups appears at the wavenumber of $1693 \mathrm{~cm}^{-1}$, and the band at around $1384 \mathrm{~cm}^{-1}$ was assigned to the stretching of carboxylate groups in heterogeneous macromolecular materials (Chen et al. 2012). The peak at around $1268 \mathrm{~cm}^{-1}$ was assigned to aromatic $\mathrm{C}-\mathrm{O}$ and phenolic $-\mathrm{OH}$ stretching. $\mathrm{C}-\mathrm{H}$ bending for aromatic out-of-plane deformation appeared at around 882,821 and $758 \mathrm{~cm}^{-1}$ (Chen et al. 2008). Biochar had a specific surface area of $85.3 \mathrm{~m}^{2} \cdot \mathrm{g}^{-1}$ and an average pore size of $2.57 \mathrm{~nm}$ (Fig. 1b). The $\mathrm{pH}_{\mathrm{pzc}}$ of biochar was estimated to be about 2.8 (Fig. 1c). Thus, the surfaces of biochar were negatively charged at $\mathrm{pH}$ values higher than 2.8 due to the dissociation of carboxyl and hydroxyl groups.

The DLS measurements showed that DHA particle sizes had a lognormal distribution with the major peak appeared at $152 \pm 15 \mathrm{~nm}$ (Fig. 2a). The AFM image (Fig. 2b) showed some irregular strip and spongelike structures, indicating formation of aggregates of DHA molecules. Moreover, some molecules with smaller apparent size can also be seen in the image. The image also showed some uniform and tiny particles, which indicated a portion of relatively uniform DHA molecules (Fig. 2b). Thus, the AFM image was consistent with DLS measurements, providing more information on conformation and structure of DHA. The negative zeta potential values ranging from -11.3 to $-17.2 \mathrm{mV}$ (Fig. 2c) indicated that DHA was negatively charged in the experimental system.

\subsection{Sorption of DHA to biochar}

Sorption data of DHA to biochar in different solutions were fitted well by the Initial Mass isotherm (Fig. 3). Addition of electrolytes facilitated the sorption of DHA. The calculated $K_{d}$ value of DHA to biochar increased by 38 and $25 \%$, respectively, after addition of $\mathrm{Na}^{+}$and $\mathrm{Ca}^{2+}$ (Fig. 3). The reason for the increased DHA sorption was assumed to arise from the changed interactions between DHA and biochar with addition of electrolytes. Sorption mechanisms of DHA to biochar mainly involved hydrogen bonding, hydrophobic and $\pi-\pi$ interactions (Bhatnagar and Sillanpää 2017; Lian et al. 2015). At experimental conditions ( $\mathrm{pH}$ 6.0), both DHA and biochar surfaces were negatively charged (Fig. 1c and Fig. 2c). Addition of $\mathrm{Na}^{+}$or $\mathrm{Ca}^{2+}$ could screen the
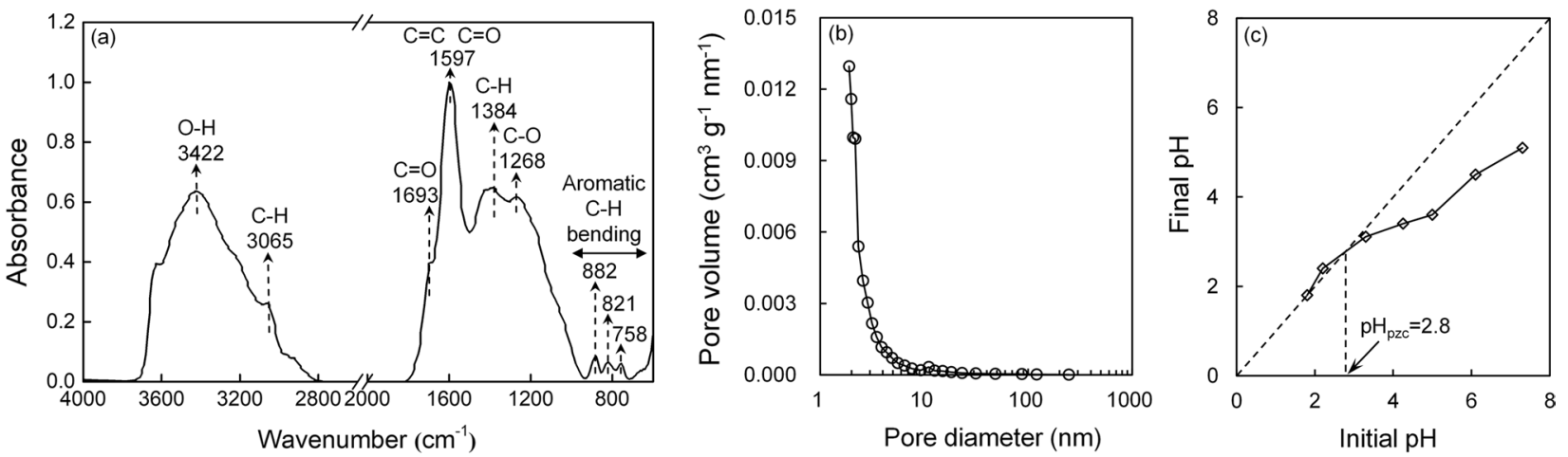

Fig. 1 FTIR spectra (a), pore size distribution (b) and determination of the $\mathrm{pH}_{\mathrm{pzc}}(\mathbf{c})$ of the biochar 

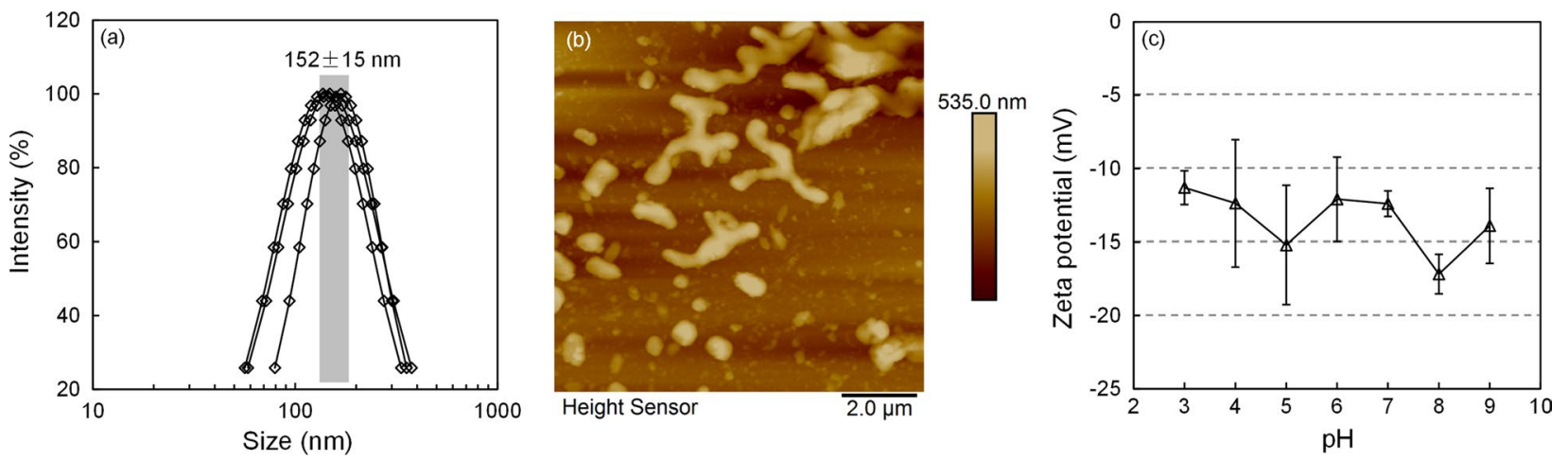

Fig. 2 Intensity-based particle size distribution (a), AFM image (b) and zeta potential (c) of DHA

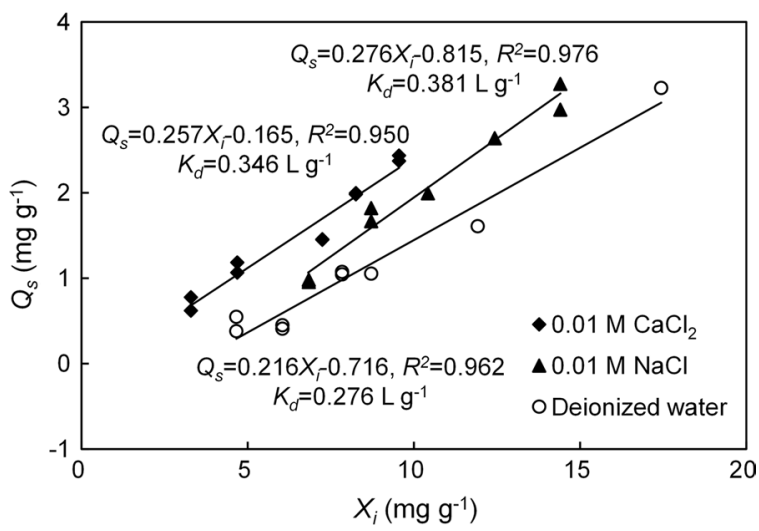

Fig. 3 Sorption isotherms of DHA to biochar in different solutions. The solid lines represent the Initial Mass isotherm fitting results

electrostatic repulsion among the segments of DHA molecules, and thus resulted in a denser DHA macromolecular structure (Pan et al. 2008). This helped DHA molecules better contact the biochar surfaces, and they can be more easily sorbed. At the same time, $\mathrm{Na}^{+}$and $\mathrm{Ca}^{2+}$ in solution could decrease the repulsive force between DHA and biochar by neutralizing the negative charges. Test results showed that the concentration of introduced $\mathrm{Na}^{+}$from $\mathrm{pH}$ adjustment in DHA solution was $300 \mathrm{mg} \cdot \mathrm{L}^{-1}$. Thus, DHA sorption to biochar in deionized water could also be facilitated by such an amount of $\mathrm{Na}^{+}$due to the fact that additional $\mathrm{Na}^{+}$enhanced DHA sorption (Fig. 3).

Lower amount of DOM was released from biochar in $\mathrm{CaCl}_{2}$ solution compared with that in $\mathrm{NaCl}$ solution and deionized water $\left(0.165\right.$ versus $0.815 / 0.716 \mathrm{mg} \mathrm{g}^{-1}$ according to the calculated $r$ values of the Initial Mass isotherm) (Fig. 3). This may be because, compared with monovalent cation $\mathrm{Na}^{+}$, bivalent cation $\mathrm{Ca}^{2+}$ bound to negatively charged biochar surfaces could serve as bridges in DOM sorption to biochar as reported in previous studies ( $\mathrm{Li}$ et al. 2017; MacKay and Canterbury 2005). It should be noted that the



Fig. 4 Sorption of BTA to DHA in different solutions determined by dialysis bag sorption experiments. Initial concentrations of DHA and BTA are 8.1 and $30 \mathrm{mg} \mathrm{L}^{-1}$, respectively

calculated amount of DOM released from biochar was less than $0.12 \%$ of the total biochar C. In addition, the added exogenous DHA amount in experimental systems was about 6.5 to 58 times higher than the released DOM amount from biochar. Thus, it is reasonable to infer that effect of released DOM from biochar on DHA sorption was limited.

\subsection{Sorption of BTA to DHA}

According to the results of dialysis bag sorption experiments (Fig. 4), sorption of BTA to DHA in $\mathrm{NaCl}$ solution showed a decrease (22\%) compared with that in deionized water. However, BTA sorption to DHA in $\mathrm{CaCl}_{2}$ solution was quite higher than that in deionized water with $K_{\mathrm{DHA}}$ value increasing by 4.52 times. DHA was proposed to be amphiphilic in nature, and it can form regions similar to detergent micelles in aqueous phase (Ragle et al. 1997; Wu et al. 2010). Under experimental conditions (pH 6.0), some carboxyl and hydroxyl functional groups of DHA were deprotonated, and the molecules were negatively charged. The molecules were stretched due to the inter- or intramolecular repulsion. But the weakly condensed aromatic rings 
with plenty of attached alicyclic and aliphatic structures can still exist as hydrophobic domain, which were also identified as discrete hydrophobic region (Pan et al. 2007). For neutral organic compounds such as BTA, hydrophobic and $\pi-\pi$ interactions with the hydrophobic domain are mainly responsible for sorption to DHA (Longstaffe et al. 2013; Pan et al. 2007; Zhu et al. 2004). In this way, sorption of BTA to DHA in deionized water was observed (Fig. 4).

The conformation of DOM is sensitive to changes of salt concentration and cation valence, which affects its interactions with organic contaminants (Pan et al. 2008; Ragle et al. 1997; Schlautman and Morgan 1993). As salts were added, the negative charges of DHA were neutralized and interand intramolecular repulsions decreased, and consequently the aggregation of DHA molecules were promoted. Though larger hydrophobic domains for BTA can be formed due to the gathering of separated hydrophobic microenvironment, the $K_{\text {DHA }}$ decreased after addition of $\mathrm{Na}^{+}$(Fig. 4). Therefore, a more compressed form of aggregates could be formed. Hence, $K_{\mathrm{DHA}}$ decreased owing to the low availability of sorption sites. The results of previous research on this subject indicated that these effects may be quite complex. Similarly, it was found that sorption of polycyclic aromatic hydrocarbons (perylene, pyrene and anthracene) by DHA generally decreased with increasing ionic strength (0.001-0.1 M NaCl) (Schlautman and Morgan 1993). The reason was mainly due to the decrease in size of hydrophobic sites, also known as hydrophobic cavities where van der Waals forces between DHA and organic compounds can happen as a portion of the whole hydrophobic interactions. Thus, the inability of organic molecules to enter the reduced-size hydrophobic cavities caused the sorption inhibition. Furthermore, it seemed unlikely that the salting-out effect would have a strong impact on BTA-DHA interactions because of the relatively low ionic strength $(0.01 \mathrm{M})$.

On the other hand, divalent cations like $\mathrm{Ca}^{2+}$ enable bridging of negatively charged groups of DHA and then cause the DHA macromolecules to coil (Pan et al. 2008). The self-coiling process and aggregation between DHA molecules can form large hydrophobic regions, leading to a more favorable partitioning and $\pi-\pi$ interaction environment (Longstaffe et al. 2013; Pan et al. 2007). Therefore, BTA sorption was remarkably enhanced (Fig. 4).

\subsection{Effect of DHA on BTA sorption}

Sorption isotherms of BTA to biochar in different solutions can be well fitted by the Dual-mode model (Fig. 5a-c and
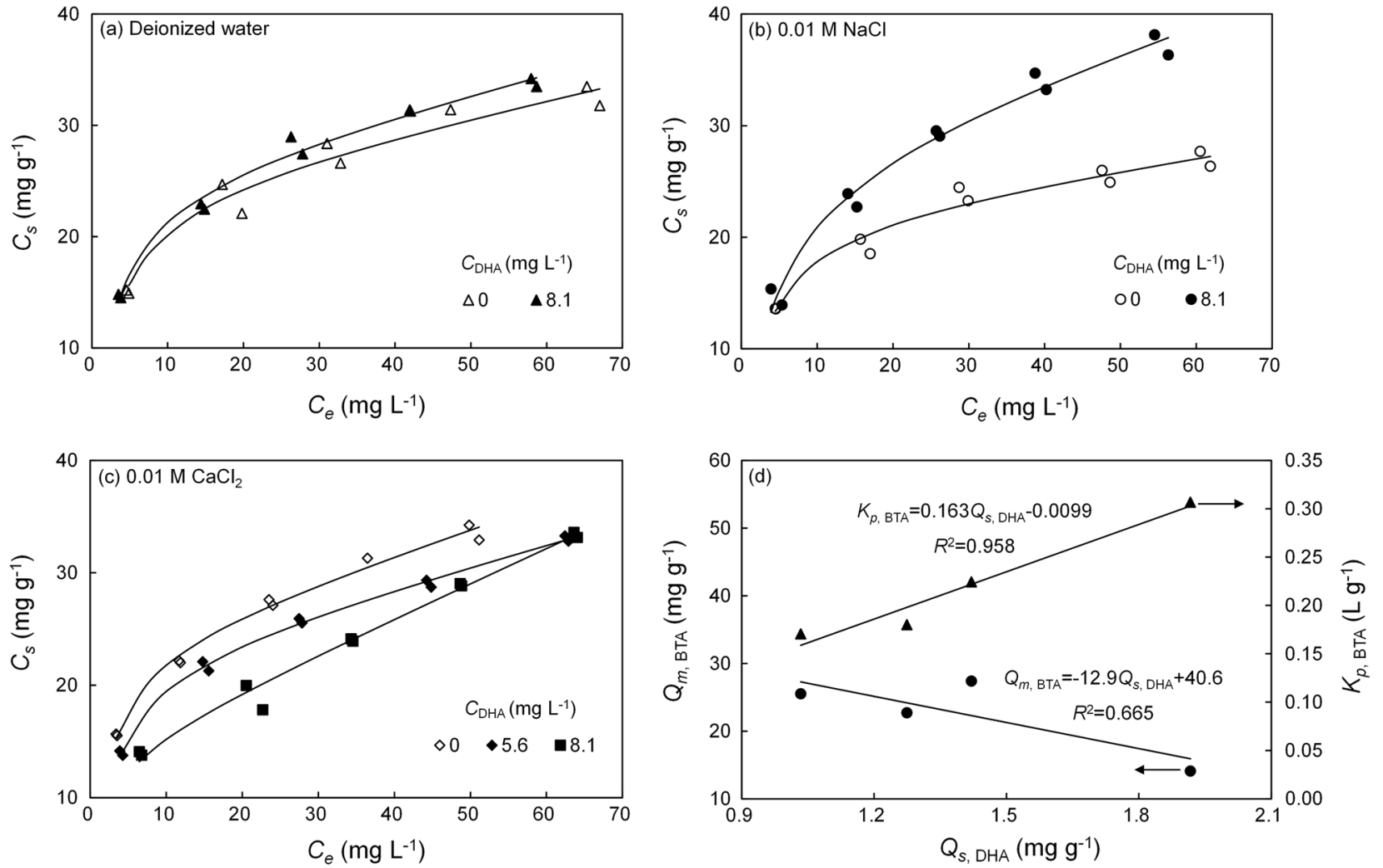

Fig. 5 Isotherms of BTA sorption to biochar in different solutions (a-c), and correlations of the calculated $K_{p}$ and $Q_{m}$ of BTA with $Q_{s}$ of DHA (d). The solid lines in panels $\mathbf{a}-\mathbf{c}$ are the Dual-mode model fitting results 
Table 1), demonstrating that both partition and adsorption contributed to the sorption. In addition, high nonlinearity of the isotherms was observed with Freundlich $n$ values varying from 0.267 to 0.381 (Table 1), especially at low BTA concentrations. The nonlinearity at low concentrations was ascribed to adsorption interactions (such as $\pi-\pi$ interactions) between BTA and biochar sites with high activity (e.g., aromatic structures) (Hart et al. 2004; Keiluweit and Kleber 2009). At moderate to high concentrations, the adsorption gradually became saturated and the partition in noncarbonized phase of biochar (Chen et al. 2008) resulted in an apparent linear isotherm.

Variations in the partition and adsorption components of biochar in different solutions were assessed using the partition coefficient $\left(K_{p}\right)$ and the maximum adsorption capacity $\left(Q_{m}\right)$ from the Dual-mode model, respectively. As can be seen in Table 1 , the $Q_{m}$ in deionized water $\left(24.8 \mathrm{mg} \mathrm{g}^{-1}\right)$ decreased with addition of $\mathrm{Na}^{+}\left(22.1 \mathrm{mg} \mathrm{g}^{-1}\right)$ or $\mathrm{Ca}^{2+}$ (23.8 $\left.\mathrm{mg} \mathrm{g}^{-1}\right)$. The $K_{p}$ in deionized water $\left(0.144 \mathrm{~L} \mathrm{~g}^{-1}\right)$ decreased with addition of $\mathrm{Na}^{+}\left(0.101 \mathrm{~L} \mathrm{~g}^{-1}\right)$ but increased with addition of $\mathrm{Ca}^{2+}\left(0.220 \mathrm{~L} \mathrm{~g}^{-1}\right)$. After addition of DHA, the $K_{p}$ increased from $0.144 \mathrm{~L} \mathrm{~g}^{-1}$ to $0.171 \mathrm{~L} \mathrm{~g}^{-1}$ in deionized water, from $0.101 \mathrm{~L} \mathrm{~g}^{-1}$ to $0.224 \mathrm{~L} \mathrm{~g}^{-1}$ in $\mathrm{NaCl}$ solution and from $0.220 \mathrm{~L} \mathrm{~g}^{-1}$ to $0.307 \mathrm{~L} \mathrm{~g}^{-1}$ in $\mathrm{CaCl}_{2}$ solution. While, the $Q_{m}$ increased from $24.8 \mathrm{mg} \mathrm{g}^{-1}$ to $25.5 \mathrm{mg} \mathrm{g}^{-1}$ in deionized water and from $22.1 \mathrm{mg} \mathrm{g}^{-1}$ to $27.4 \mathrm{mg} \mathrm{g}^{-1}$ in $\mathrm{NaCl}$ solution, but decreased from $23.8 \mathrm{mg} \mathrm{g}^{-1}$ to $14.1 \mathrm{mg} \mathrm{g}^{-1}$ in $\mathrm{CaCl}_{2}$ solution. These observations indicated that partition and adsorption of BTA to biochar changed with variation in solution chemistry (such as different cations and DHA). The phenomenon can be attributed to the following reasons.

As the biochar surfaces were negatively charged at experimental conditions ( $\mathrm{pH}$ 6.0), they had a strong

Table 1 Sorption parameters of BTA to biochar fitted by different isotherm models

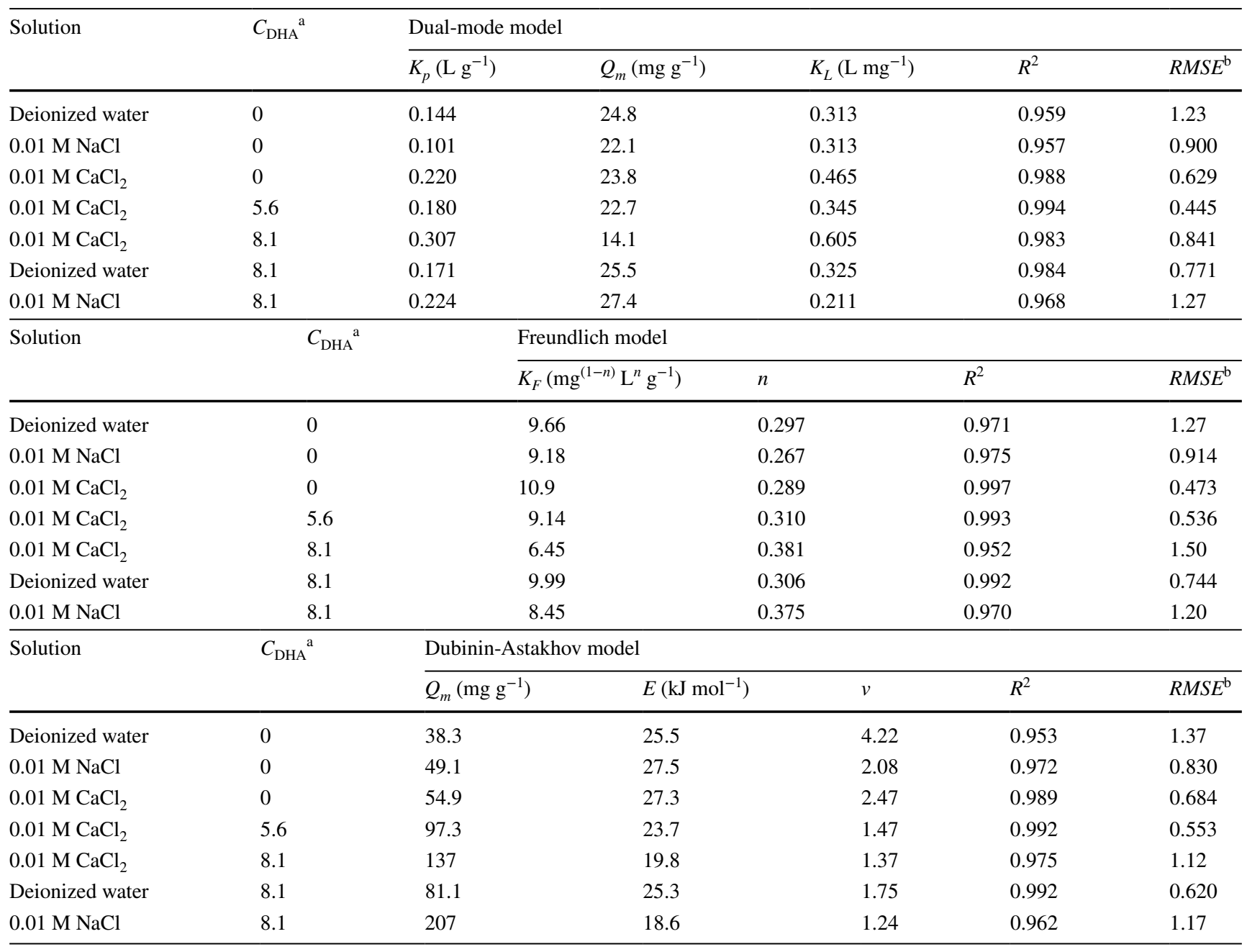

${ }^{\mathrm{a}} \mathrm{DHA}$ concentration $\left(\mathrm{mg} \cdot \mathrm{L}^{-1}\right)$

${ }^{\mathrm{b}} R M S E$ (root mean squared error) $=\operatorname{SQRT}\left(\left(\sum_{i=1}^{N}\left(C_{s, f i t t e d}-C_{s, \text { measured }}\right)^{2}\right) / N\right), N$ is the number of data points 
appetency for the added $\mathrm{Na}^{+}$or $\mathrm{Ca}^{2+}$ by electrostatic attraction. As a result, the biochar's exterior was covered by the cations. Hence, the hydration of cations could block BTA access to sites on biochar surfaces (Chen et al. 2007), leading to a slight decrease in $Q_{m}$ of BTA. The variation in $K_{p}$ after addition of cations probably resulted from the potential altered partition domains as described above.

Several observations for effect of DOM on sorption of organic pollutants by biochar have been reported, including competition for adsorption sites, pore blockage by DOM coating and provision of extra sorption sites or partitioning phase for organic pollutants (Lian et al. 2015; Wu et al. 2018a; Zhang et al. 2015). The increased $K_{p}$ after addition of DHA was directly attributed to enhanced partitioning of BTA into DHA covered on biochar, which was supported by the observed results of BTA sorption to DHA (Fig. 4). Since the sorbed amount of DHA to biochar apparently increased in $\mathrm{CaCl}_{2}$ solution (Fig. 3), the decreased $Q_{m}$ of BTA in $\mathrm{CaCl}_{2}$ solution after addition of DHA can be due to the competition of DHA for sorption sites and/or pore blockage by DHA coating. As a matter of fact, the size at major peak of DHA particle size distribution was remarkably higher than the average pore size of biochar $(152 \pm 15 \mathrm{~nm}$ versus $2.57 \mathrm{~nm})$ (Figs. $1 \mathrm{~b}$ and 2a). Thus, the competition and pore blockage from DHA decreased the availability of sites for BTA, leading to the inhibitory role of DHA in BTA adsorption to biochar. This was further proved by the reduction in $K_{F}$ and $E$ values from Freundlich and Dubinin-Astakhov models, respectively (Table 1), demonstrating the decreased sorption affinity of BTA due to DHA sorption to biochar. The adsorption interaction of BTA with DHA could be neglected when compared with its sorption to biochar. The slightly increased $Q_{m}$ of BTA in deionized water and $\mathrm{NaCl}$ solution after addition of DHA was presumably due to the higher exposed surface area after dispersion by DHA (Pan et al. 2013). Similarly, this effect was considered to cause the slightly increased sulfamethoxazole sorption to biochar at low DHA concentrations $(<10 \mathrm{mg}$ $\mathrm{L}^{-1}$ ) (Lian et al. 2015).

To further verify the mechanisms, the sorbed amount $\left(Q_{s}\right)$ of DHA to biochar was calculated according to the Initial Mass isotherm parameters (Fig. 3), then potential relationships of $K_{p}$ and $Q_{m}$ of BTA with $Q_{s}$ of DHA were examined. From Fig. 5d, a strong positive correlation between $K_{p}$ of BTA and $Q_{s}$ of DHA, and a negative correlation between $Q_{m}$ of BTA and $Q_{s}$ of DHA were observed. The results further confirmed that the sorbed DHA on biochar can not only serve as partitioning phase to promote the partition of BTA but also compete for sites and/or block pores available for BTA to inhibit its adsorption. The variation in apparent BTA sorption to biochar affected by DHA was thus a combination of changes in both its partition and adsorption properties.

\subsection{Site energy distribution analysis}

Identifying site energy distribution of BTA sorption to biochar can provide useful information on sorption mechanisms. In both DHA-free and DHA-BTA systems, $E^{*}$ decreased sharply with an increase of BTA loading (Fig. 6a-c), which could be understood by the fact that BTA occupied the high-energy sorption sites of biochar first, and then transferred to the low-energy sorption sites. In this experimental system, the high-energy sorption sites of biochar for BTA can be the aromatic structures allowing specific interactions (such as $\pi-\pi$ interactions) as discussed above. The noncarbonized phase of biochar was considered as the low-energy sorption sites for partition. This was supported by the calculation based on the Dual-mode model fitting results (data not shown) that contribution of adsorption to the total sorption decreased while that of partition increased with an increase of BTA loading.

The site energy distribution curves of BTA sorption with and without DHA addition showed similar features (Fig. 6d-f). With the increase of $E^{*}, F\left(E^{*}\right)$ increased to reach a maximum, and then gradually decreased to near zero. This demonstrated that BTA was primarily adsorbed to sites with high energy at low concentrations and interacted with sites with low energy at high concentrations. However, distinct differences between the energy distribution with and without DHA addition were also observed. The peak of site energy distribution curves was indicated as $F\left(E_{0} *\right)$, meanwhile, the site energy $\left(E_{0}{ }^{*}\right)$ at the peak position showed the highest occurring frequency. The two parameters can provide valuable information in describing solute-sorbent interactions ( $\mathrm{Li}$ et al. 2021; Shen et al. 2015). The energy distribution curve of BTA sorption with DHA addition showed lower $E_{0} *$ (3.41-10.4 versus $\left.13.1-20.1 \mathrm{~kJ} \mathrm{~mol}^{-1}\right)$ and higher $F\left(E_{0}^{*}\right)(4.09-16.1$ versus 2.35-2.88 $\mathrm{mol} \mathrm{mg} \mathrm{kJ}^{-1} \mathrm{~g}^{-1}$ ) than those without DHA addition (Fig. 6d-f). Therefore, BTA tended to occupy more toward the relatively low-energy sites with the coexistence of DHA. While for the none-DHA system, BTA tended to occupy more toward the relatively high-energy sites. This was also consistent with the DHA effects on BTA sorption as identified by the above interpretation because DHA can provide additional partitioning phase (low-energy sites) for BTA but decrease the high-energy sites by competing for adsorption sites and/or blocking pores available for BTA. As the sorption energies of adsorption and partition were different, the same $E^{*}$ for BTA sorption with and without DHA addition may not reliably indicate the same sites on biochar. Therefore, quantitative analysis on the 

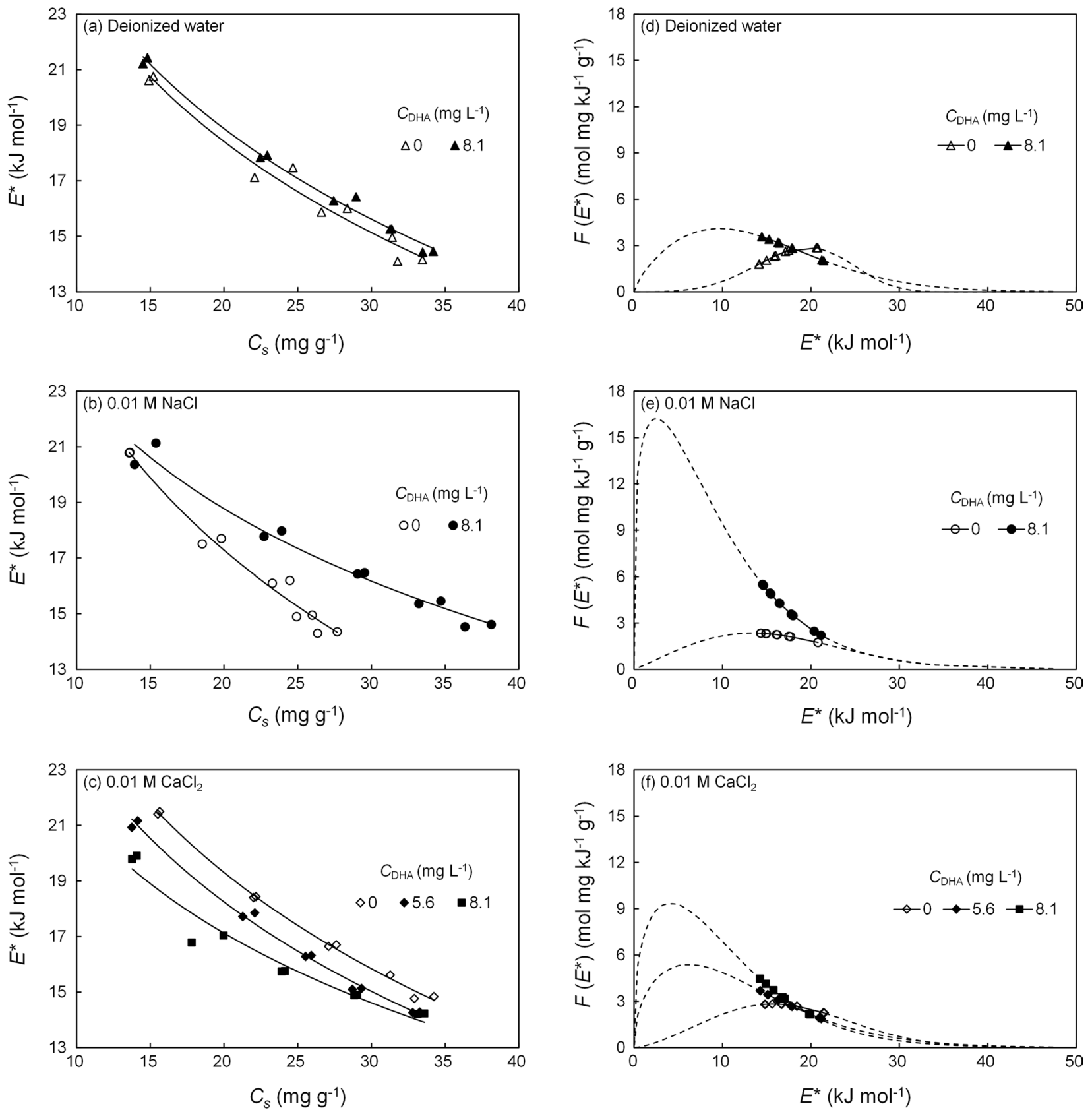

Fig. 6 Dependence of site energy on BTA loading (a-c) and site energy distribution of BTA (d-f). In panels d-f, solid lines denote site energy distribution within the range of experimental data and dashed lines denote site energy distribution beyond the range of experimental data

energy distribution was averted and only the variation of $E_{0} *$ affected by DHA was assessed.

\subsection{Prediction of BTA sorption affected by DHA}

Due to the fact that DHA can play diverse roles in partition (promoted) and adsorption (inhibited) of BTA to biochar as stated before, an improved model (Eq. (11)) modified from the Dual-mode model was put forward to better describe BTA sorption affected by DHA according to the observed relationships (Fig. 5d).

$C_{s}=\left(a C_{\mathrm{DHA}}+1\right) K_{p} C_{e}+\left(b C_{\mathrm{DHA}}+1\right) Q_{m} K_{L} C_{e} /\left(1+K_{L} C_{e}\right)$

where $a$ and $b$ indicate fitting parameters under the DHA effect.

The Microsoft Excel Solver tool was used to calculate the parameters in Eq. (11) according to the sorption data 


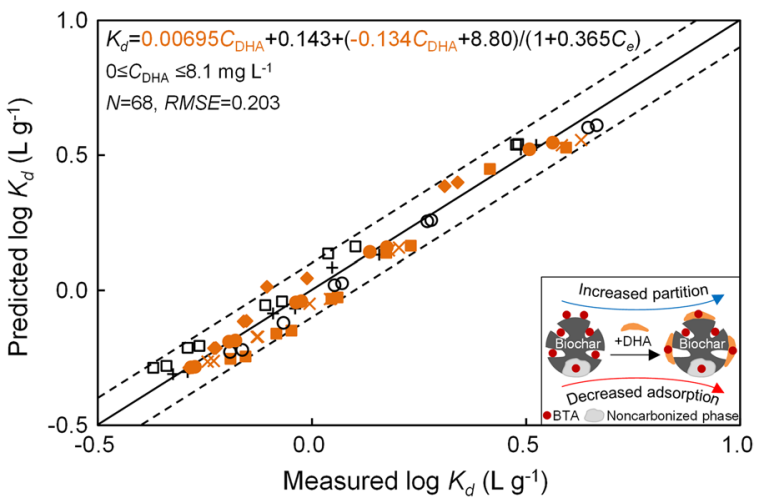

Fig. 7 Comparison between the predicted and measured $\log K_{d}$ values of BTA in different solutions $\left[\mathrm{H}_{2} \mathrm{O}(+), \mathrm{H}_{2} \mathrm{O}+\right.$ DHA $8.1 \mathrm{mg} \mathrm{L}^{-1}(\times), \mathrm{NaCl}$ (口), $\mathrm{NaCl}+\mathrm{DHA} 8.1 \mathrm{mg} \mathrm{L}^{-1}(\square), \mathrm{CaCl}_{2}(\mathrm{O}), \mathrm{CaCl}_{2}+\mathrm{DHA} 5.6 \mathrm{mg} \mathrm{L}^{-1}$ $(\bullet)$, and $\left.\mathrm{CaCl}_{2}+\mathrm{DHA} 8.1 \mathrm{mg} \mathrm{L}^{-1}(\diamond)\right]$. The solid line indicates the $1: 1$ line, and dashed lines denote $0.1 \log$ unit deviations. RMSE (root mean squared error $)=\operatorname{SQRT}\left(\left(\sum_{i=1}^{N}\left(K_{d, \text { fitted }}-K_{d \text {,measured }}\right)^{2}\right) / N\right)$, and $N$ is the number of data points

in Fig. 5a-c. Subsequently, the predicted $K_{d}$ values of BTA were derived from Eq. (6) and then compared to the experimental values. It can be seen from Fig. 7 that the proposed model displayed a good performance in describing BTA sorption to biochar under the DHA effect. Specifically, most deviations between the measured and predicted $K_{d}$ values were less than $0.1 \log$ unit. Thus, the proposed model can be used as an effective way in predicting sorption of BTA to biochar in different solutions affected by DHA.

\section{Conclusions}

This study emphasized that DHA can pose diverse roles in partition and adsorption of BTA to biochar. On the one hand, adsorption of BTA was inhibited by DHA due to its competition for sites and/or pore blockage of biochar. On the other hand, partition of BTA was promoted by DHA since the sorbed DHA on biochar can provide additional partitioning phase for BTA. This was verified by the site energy distribution analysis of BTA sorption that the site energy of the highest occurring frequency decreased from 13.1-20.1 to $3.41-10.4 \mathrm{~kJ} \mathrm{~mol}^{-1}$ with addition of DHA. Sorption of BTA to biochar in different electrolyte solutions affected by DHA can be well predicted by an improved Dual-mode model including the aqueous concentration of DHA, with most $K_{d}$ $\left(\mathrm{L} \mathrm{g}^{-1}\right)$ deviations within $0.1 \log$ unit. Further studies will be required to evaluate the above discussed concept to other contaminants, sorbents with different degrees of carbonization, and DOM (e.g., different fractions extracted from natural soils) to better understand the organic contaminantDOM-biochar interactions.
Acknowledgements This work was supported by the National Natural Science Foundation of China (41172223 and 41902259) and the Natural Science Foundation of Hebei Province of China (D2020504006).

Authors' contributions Lin $\mathrm{Wu}$ : formal analysis, writing-original draft, writing - review \& editing, and visualization. Xiaoli Zhao: conceptualization, methodology, investigation, and data curation. Erping Bi: supervision, project administration, and funding acquisition.

Availability of data and materials All data generated or analysed during this study are included in this published article.

Code availability Not applicable.

\section{Declarations}

Ethical approval Not applicable.

Consent to participate Not applicable.

Consent for publication Not applicable.

Conflict of interest The authors declare no conflict of interest.

Open Access This article is licensed under a Creative Commons Attribution 4.0 International License, which permits use, sharing, adaptation, distribution and reproduction in any medium or format, as long as you give appropriate credit to the original author(s) and the source, provide a link to the Creative Commons licence, and indicate if changes were made. The images or other third party material in this article are included in the article's Creative Commons licence, unless indicated otherwise in a credit line to the material. If material is not included in the article's Creative Commons licence and your intended use is not permitted by statutory regulation or exceeds the permitted use, you will need to obtain permission directly from the copyright holder. To view a copy of this licence, visit http://creativecommons.org/licenses/by/4.0/.

\section{References}

Accardi-Dey A, Gschwend PM (2003) Reinterpreting literature sorption data considering both absorption into organic carbon and adsorption onto black carbon. Environ Sci Technol 37:99-106

Alotaibi MD, McKinley AJ, Patterson BM, Reeder AY (2015) Benzotriazoles in the aquatic environment: a review of their occurrence, toxicity, degradation and analysis. Water Air Soil Pollut 226:226

Bhatnagar A, Sillanpää M (2017) Removal of natural organic matter (NOM) and its constituents from water by adsorption - a review. Chemosphere 166:497-510

Bi E, Schmidt TC, Haderlein SB (2007) Environmental factors influencing sorption of heterocyclic aromatic compounds to soil. Environ Sci Technol 41:3172-3178

Breedveld GD, Roseth R, Sparrevik M, Hartnik T, Hem LJ (2003) Persistence of the de-icing additive benzotriazole at an abandoned airport. Water Air Soil Pollut 3:91-101

Carter MC, Kilduff JE, Weber WJ (1995) Site energy distribution analysis of preloaded adsorbents. Environ Sci Technol 29:1773-1780

Chen J, Zhu D, Sun C (2007) Effect of heavy metals on the sorption of hydrophobic organic compounds to wood charcoal. Environ Sci Technol 41:2536-2541 
Chen B, Zhou D, Zhu L (2008) Transitional adsorption and partition of nonpolar and polar aromatic contaminants by biochars of pine needles with different pyrolytic temperatures. Environ Sci Technol 42:5137-5143

Chen Z, Chen B, Chiou CT (2012) Fast and slow rates of naphthalene sorption to biochars produced at different temperatures. Environ Sci Technol 46:11104-11111

El-Naggar A, Lee SS, Rinklebe J, Farooq M, Song H, Sarmah AK, Zimmerman AR, Ahmad M, Shaheen SM, Ok YS (2019) Biochar application to low fertility soils: a review of current status, and future prospects. Geoderma 337:536-554

Engel M, Chefetz B (2016) Removal of triazine-based pollutants from water by carbon nanotubes: impact of dissolved organic matter (DOM) and solution chemistry. Water Res 106:146-154

Hart DS, Davis LC, Erickson LE, Callender TM (2004) Sorption and partitioning parameters of benzotriazole compounds. Microchemical J 77:9-17

He T, Zhang T, Liu S, Shi J, Huang Y, Zheng H, Liu W (2019) Toxicological effects benzotriazole to the marine scallop Chlamys nobilis: a 2-month exposure study. Environ Sci Pollut Res 26:10306-10318

Jia Y, Aagaard P, Breedveld GD (2007) Sorption of triazoles to soil and iron minerals. Chemosphere 67:250-258

Jin J, Kang M, Sun K, Pan Z, Wu F, Xing B (2016) Properties of biochar-amended soils and their sorption of imidacloprid, isoproturon, and atrazine. Sci Total Environ 550:504-513

Kah M, Sigmund G, Xiao F, Hofmann T (2017) Sorption of ionizable and ionic organic compounds to biochar, activated carbon and other carbonaceous materials. Water Res 124:673-692

Kahle M, Buerge IJ, Müller MD, Poiger T (2009) Hydrophilic anthropogenic markers for quantification of wastewater contamination in ground-and surface waters. Environ Toxicol Chem 28:2528-2536

Kalbitz K, Solinger S, Park J-H, Michalzik B, Matzner E (2000) Controls on the dynamics of dissolved organic matter in soils: a review. Soil Sci 165:277-304

Keiluweit M, Kleber M (2009) Molecular-level interactions in soils and sediments: the role of aromatic $\pi$-systems. Environ Sci Technol 43:3421-3429

Keiluweit M, Nico PS, Johnson MG, Kleber M (2010) Dynamic molecular structure of plant biomass-derived black carbon (biochar). Environ Sci Technol 44:1247-1253

Kowalska K, Felis E, Sochacki A, Bajkacz S (2019) Removal and transformation pathways of benzothiazole and benzotriazole in membrane bioreactors treating synthetic municipal wastewater. Chemosphere 227:162-171

Li M, Zhang A, Wu H, Liu H, Lv J (2017) Predicting potential release of dissolved organic matter from biochars derived from agricultural residues using fluorescence and ultraviolet absorbance. J Hazard Mater 334:86-92

Li Y, Bi E, Chen H (2019) Effects of dissolved humic acid on fluoroquinolones sorption and retention to kaolinite. Ecotoxicol Environ Saf 178:43-50

Li X, Lei F, Li B, Bi E (2021) Different binding characteristics of ciprofloxacin to iron mineral surfaces: thermodynamic evidence and site energy distribution analysis. J Environ Qual 50:706-716

Lian F, Sun B, Chen X, Zhu L, Liu Z, Xing B (2015) Effect of humic acid (HA) on sulfonamide sorption by biochars. Environ Pollut 204:306-312

Liang X, Zha J, Martyniuk CJ, Wang Z, Zhao J (2017) Histopathological and proteomic responses in male Chinese rare minnow (Gobiocypris rarus) indicate hepatotoxicity following benzotriazole exposure. Environ Pollut 229:459-469

Longstaffe JG, Courtier-Murias D, Simpson AJ (2013) The pH-dependence of organofluorine binding domain preference in dissolved humic acid. Chemosphere 90:270-275
Lu L, Yu W, Wang Y, Zhang K, Zhu X, Zhang Y, Wu Y, Ullah H, Xiao X, Chen B (2020) Application of biochar-based materials in environmental remediation: from multi-level structures to specific devices. Biochar 2:1-31

MacKay AA, Canterbury B (2005) Oxytetracycline sorption to organic matter by metal-bridging. J Environ Qual 34:1964-1971

Nodvin SC, Driscoll CT, Likens GE (1986) Simple partitioning of anions and dissolved organic carbon in a forest soil. Soil Sci 142:27-35

Pan B, Ghosh S, Xing B (2007) Nonideal binding between dissolved humic acids and polyaromatic hydrocarbons. Environ Sci Technol 41:6472-6478

Pan B, Ghosh S, Xing B (2008) Dissolved organic matter conformation and its interaction with pyrene as affected by water chemistry and concentration. Environ Sci Technol 42:1594-1599

Pan B, Zhang D, Li H, Wu M, Wang Z, Xing B (2013) Increased adsorption of sulfamethoxazole on suspended carbon nanotubes by dissolved humic acid. Environ Sci Technol 47:7722-7728

Ragle CS, Engebretson RR, von Wandruszka R (1997) The sequestration of hydrophobic micropollutants by dissolved humic acids. Soil Sci 162:106-114

Schlautman MA, Morgan JJ (1993) Effects of aqueous chemistry on the binding of polycyclic aromatic hydrocarbons by dissolved humic materials. Environ Sci Technol 27:961-969

Shen X, Guo X, Zhang M, Tao S, Wang X (2015) Sorption mechanisms of organic compounds by carbonaceous materials: site energy distribution consideration. Environ Sci Technol 49:4894-4902

Shi Z, Liu Y, Xiong Q, Cai W, Ying G (2019) Occurrence, toxicity and transformation of six typical benzotriazoles in the environment: a review. Sci Total Environ 661:407-421

Wagner TV, Parsons JR, Rijnaarts HHM, de Voogt P, Langenhoff AAM (2020) Benzotriazole removal mechanisms in pilot-scale constructed wetlands treating cooling tower water. J Hazard Mater 384:121314

Wu W, Sun H, Wang L, Li K, Wang L (2010) Comparative study on the micelle properties of synthetic and dissolved organic matters. J Hazard Mater 174:635-640

Wu L, Yang N, Li B, Bi E (2018a) Roles of hydrophobic and hydrophilic fractions of dissolved organic matter in sorption of ketoprofen to biochars. Environ Sci Pollut Res 25:31486-31496

Wu W, Sheng H, Gu C, Song Y, Willbold S, Qiao Y, Liu G, Zhao W, Wang Y, Jiang X, Wang F (2018b) Extraneous dissolved organic matter enhanced adsorption of dibutyl phthalate in soils: insights from kinetics and isotherms. Sci Total Environ 631-632:1495-1503

Wu L, Dai JE, Bi E (2020a) Roles of dissolved humic acid and tannic acid in sorption of benzotriazole to a sandy loam soil. Ecotoxicol Environ Saf 204:111088

Wu P, Wang Z, Wang H, Bolan NS, Wang Y, Chen W (2020b) Visualizing the emerging trends of biochar research and applications in 2019: a scientometric analysis and review. Biochar 2:135-150

Xiao L, Bi E, Du B, Zhao X, Xing C (2014) Surface characterization of maize-straw-derived biochars and their sorption performance for MTBE and benzene. Environ Earth Sci 71:5195-5205

Xing C, Yu C, Bi E, Dai J (2016) Interactions between benzotriazole and corncob biochars pyrolyzed at different temperature. J Groundwater Sci Eng 4:320-327

Xu B, Wu F, Zhao X, Liao H (2010) Benzotriazole removal from water by Zn-Al-O binary metal oxide adsorbent: behavior, kinetics and mechanism. J Hazard Mater 184:147-155

Yang Y, Chun Y, Sheng G, Huang M (2004) pH-dependence of pesticide adsorption by wheat-residue-derived black carbon. Langmuir 20:6736-6741

Zhang Z, Ren N, Li Y-F, Kunisue T, Gao D, Kannan K (2011) Determination of benzotriazole and benzophenone UV filters in sediment and sewage sludge. Environ Sci Technol 45:3909-3916 
Zhang D, Pan B, Wu M, Zhang H, Peng H, Ning P, Xing B (2012) Cosorption of organic chemicals with different properties: their shared and different sorption sites. Environ Pollut 160:178-184

Zhang M, Shu L, Guo X, Shen X, Zhang H, Shen G, Wang B, Yang Y, Tao S, Wang X (2015) Impact of humic acid coating on sorption of naphthalene by biochars. Carbon 94:946-954
Zhu D, Hyun S, Pignatello JJ, Lee LS (2004) Evidence for $\pi-\pi$ electron donor-acceptor interactions between $\pi$-donor aromatic compounds and $\pi$-acceptor sites in soil organic matter through $\mathrm{pH}$ effects on sorption. Environ Sci Technol 38:4361-4368 